\title{
HIV infections acquired through heterosexual intercourse in the United Kingdom: findings from national surveillance
}

\author{
Sarah Dougan, Victoria L Gilbart, Katy Sinka, Barry G Evans
}

The trends underlying the rapid and substantial increases in HIV diagnoses among heterosexual people in the United Kingdom are complex and sometimes misunderstood. Although the number of people becoming infected with HIV through heterosexual intercourse in the United Kingdom is rising steadily, most of the overall rise in HIV diagnoses among heterosexuals is among people who originate from and were infected abroad, mainly in Africa. ${ }^{1}$ We describe HIV diagnoses where infection was probably acquired through heterosexual intercourse in the United Kingdom.

\section{Methods, participants, and results}

The Health Protection Agency Centre for Infections receives voluntary and confidential reports of HIV diagnoses in England, Wales, and Northern Ireland. ${ }^{2}$ For all reports, the centre collects the probable route of infection and the probable country of infection for patients who were infected through heterosexual intercourse. A research nurse/counsellor follows up where data are incomplete. Where more than one possible country of infection is given, the country with the highest prevalence of HIV is allocated by an epidemiologist or a research nurse/counsellor. If an individual was probably infected in the United Kingdom, further information concerning the partner's probable route and country of infection are recorded. If it is uncertain but possible that the partner was infected in the United Kingdom, the partner's country of infection will be assigned to "unspecified European countries." Individuals infected through heterosexual intercourse in the United Kingdom by a "high risk" partner (for example, an injecting drug user) are excluded.

Reports indicate that 21115 adults (aged 15 or older) diagnosed with HIV in England, Wales, and Northern Ireland between 1985 and 2003 were infected through heterosexual intercourse (figure). Of these, $1901(9.0 \%)$ were probably infected in the United Kingdom: in 1999, 144 such diagnoses were made, rising to 315 in 2003 . Of the 1901, the median age at diagnosis was 30 years for women and 36 years for men. Sixty two per cent (1179) were probably infected by a heterosexual partner infected outside Europe, 30\% (569) by a partner infected in Europe (including the United Kingdom), and for 8\% (153), the partner's country of infection was not established.

Of the 1179 individuals infected in the United Kingdom by a partner infected outside Europe, 75\% (882) were women, and, of the 1006 for whom this information was reported, $44 \%$ (446) were white and 34\% (341) black African. Of partners, 77\% (912) were probably infected in Africa, 16\% (194) in Latin America and the Caribbean, and 6\% (73) elsewhere.

Of the 569 individuals whose partner had been infected in Europe, $47 \%$ (269) were women, and, of the
486 individuals for whom this information was reported, $81 \%$ (393) were white. Forty per cent (230) of partners were probably infected in the United Kingdom, 6\% (34) in other identified European countries, and 54\% (305) in unspecified European countries.

\section{Comment}

Numbers of HIV infections acquired through heterosexual intercourse in the United Kingdom have risen in recent years but continue to represent a small proportion $(<10 \%)$ of all HIV infections diagnosed in heterosexuals in England, Wales, and Northern Ireland each year. Homosexual men remain at greatest risk of acquiring HIV in the United Kingdom, accounting for an estimated $80 \%$ of newly diagnosed infections that were probably acquired in the United Kingdom. ${ }^{13}$ Of those infected through heterosexual intercourse in the United Kingdom, 62\% (1179) had a sexual partner who was infected outside Europe, and nearly a third in Europe, including the United Kingdom.

This article was posted on bmj.com on 11 March 2005: http://bmj.com/ cgi/doi/10.1136/bmj.38393.572188.EB

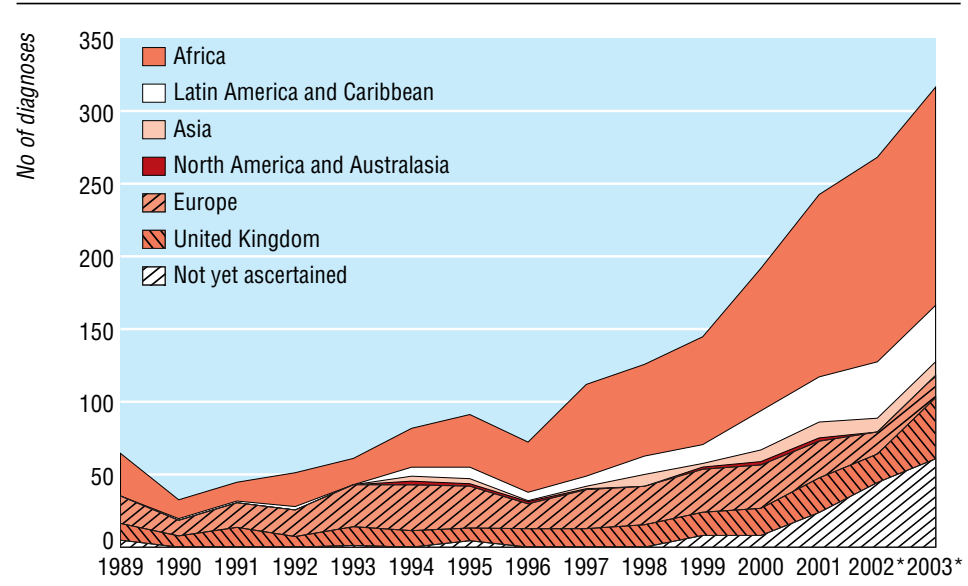

Year of earliest HIV diagnosis in England, Wales, and Northern Ireland

${ }^{*}$ Numbers will increase for more recent years as further reports are received; numbers of "not yet ascertained" will also decrease as follow up is completed

New diagnoses of HIV infection acquired through sex between men and women in the United Kingdom (excludes individuals infected through sex between men and women in the United Kingdom by a "high risk" partner (partner infected through injecting drug use, sex between men, blood transfusion, or blood products)), by year of diagnosis and sexual partner's probable region of infection. Reports received by end of June 2004, diagnoses in adults (aged 15 or older) reported in England, Wales, and Northern Ireland. Scottish data are not included as the reporting system run by the Scottish Centre for Infections and Environmental Health (SCIEH) differs in respect to follow up of infections acquired through heterosexual intercourse. (United Kingdom is used throughout to refer to country of infection, as the surveillance system records the information at this level) 
Voluntary surveillance systems are subject to under-reporting. Figures may underestimate true numbers. Miscategorisation of probable country of infection by presuming the country with the highest prevalence as the likely country of infection will underestimate the number of infections acquired in the United Kingdom, particularly among people originating from countries with high prevalence. New HIV diagnoses do not represent new HIV infections, as diagnosis can occur at any point between infection and death, which in the natural course of infection is typically 10-12 years. Furthermore, surveillance reports do not distinguish between partners infected in high prevalence countries while visiting and partners infected before migrating from those countries.

The number of people becoming infected with HIV through heterosexual intercourse in the United Kingdom is rising steadily. As the number of heterosexuals living with HIV (diagnosed and undiagnosed) in the United Kingdom grows, the likelihood of heterosexual transmission within the country will increase, particularly among ethnic minorities.

The continuing collaboration of those who contribute to the voluntary HIV/AIDS reporting system in England, Wales and Northern Ireland is gratefully acknowledged, as is the help, advice, and support of Kevin Fenton (Health Protection Agency (HPA)), Noel Gill (HPA), Phillip Mortimer (HPA), Linda Lazarus (Department of Health), and Daniel Thomas (Communicable Disease Surveillance Centre Wales), and administrative support provided by Fay Peyman (HPA) and Fateha Begum (HPA). Contributors: VLG followed up reports. SD analysed surveillance data and wrote the first draft. VLG, SD, KS, and BGE were involved in the drafting of subsequent versions. BGE is guarantor.

Competing interests: None declared.

Ethical approval: No patients' names are collected; instead surname Soundex codes are used and strict confidentiality of the data is maintained. The voluntary reporting system has approval under the section 60 regulations of the Health and Social Care Act (Statutory Instrument 1438-June 2002).

1 The UK Collaborative Group for HIV and STI Surveillance. Focus on prevention. HIV and other sexually transmitted infections in the United Kingdom in 2003. London: Health Protection Agency Centre for Infections, November 2004.

2 Public Health Laboratory Service Communicable Disease Surveillance Centre, Institute of Child Health (London), Scottish Centre for Infection and Environmental Health. HIV and AIDS in the UK. An epidemiological review: 2000. London: PHL S CDSC, 2001.

3 Health Protection Agency, Scottish Centre for Infection and Environmental Health, Information Statistics Department Scotland, Nationa Public Health Service for Wales, Communicable Disease Surveillance Centre Northern Ireland, and the Unlinked Anonymous Surveys Steering Group. Renewing the focus. HIV and other sexually transmitted infections in the United Kingdom in 2002. London: Health Protection Agency, 2003.

doi 10.1136/bmj.38393.572188.EB

\title{
RESEARCH POINTERS
}

\section{Metformin and reduced risk of cancer in diabetic patients}

\author{
Josie M M Evans, Louise A Donnelly, Alistair M Emslie-Smith, Dario R Alessi, Andrew D Morris
}

Division of

Community Health

Sciences, Section of

Public Health,

University of

Dundee, Dundee

DD2 4BF

Josie M M Evans

lecturer in

epidemiology

Louise A Donnelly

statistician

Mill Practice,

Dundee

Alistair M

Emslie-Smith

principal in general

practice

School of Life

Sciences, University

of Dundee

Dario R Alessi

principal investigator

Division of

Medicine,

University of

Dundee

Andrew D Morris

professor of diabetic

medicine

Correspondence to:

J M M Evans

j.m.m.stansfield@

dundee.ac.uk

BMJ 2005;330:1304-5
Metformin, widely given to patients with type 2 diabetes, works by targeting the enzyme AMPK (AMP activated protein kinase), which induces muscles to take up glucose from the blood. A recent breakthrough has found the upstream regulator of AMPK to be a protein kinase known as LKB $1 .{ }^{12} \mathrm{LKB} 1$ is a well recognised tumour suppressor. Activation of AMPK by metformin and exercise requires LKB1, and this would also explain why exercise is beneficial in the primary and secondary prevention of certain cancers. ${ }^{3}$ We hypothesise that metformin use in patients with type 2 diabetes may reduce their risk of cancer.

\section{Participants, methods, and results}

We tested this hypothesis using record linkage databases developed in Tayside, Scotland: a diabetes clinical information system (DARTS) and a database of dispensed prescriptions (MEMO). ${ }^{4}$ We did a pilot case-control study using previously validated methods. ${ }^{5}$

From 314127 people who were resident (or died) in Tayside in 1993-2001, 11876 had been newly diagnosed with type 2 diabetes. Of these, 923 were subsequently admitted to hospital with an ICD-9 or ICD-10 (international classification of diseases, 9 th or 10 th revision) diagnostic code for malignant cancer in study period (for which first admission occurred at least one year after diagnosis of diabetes). The index date of these cases was the date of first admission. We generated random controls from the diabetic population (two for each case); patients without cancer matched for age, year of diagnosis, and sex, and we gave them matching index dates.

\section{What this paper suggests}

Metformin may reduce the risk of cancer in patients with type 2 diabetes

\section{What research is needed now}

A more rigorous cohort study, before experimental work is initiated

We collated information about use of metformin for all cases and controls and calculated unadjusted odds ratios using conditional logistic regression (taking matching into account). The proportions of cases and controls for whom confounding data were available were smoking $73 \%$, body mass index $62 \%$, blood pressure $67 \%$, and postcode rank for material deprivation $99 \%$. We categorised continuous variables into quartile groups, with missing values forming a separate category. We adjusted odds ratios for these possible confounders.

More than half of the patients with cancer $(488 ; 53 \%)$ were men. Mean age was 73 (standard deviation 9.8) years and mean duration of diabetes was 8.5 (6.4) years. More than a third $(336 ; 36.4 \%)$ of the cases had been given at least one prescription for metformin in the year before their index date compared with 732 (39.7\%) of the controls. The unadjusted odds ratio was $0.86(95 \%$

This article was posted on bmi.com on 22 April 2005: http://bmj.com/cgi/doi/10.1136/bmj.38415.708634.F7 